
August 1934

\title{
DEPENDENCE OF SOUND ABSORPTION UPON THE AREA AND DISTRIBUTION OF THE ABSORBENT MATERIAL
}

\author{
By V. L. Chrisler
}

\section{ABSTRACT}

This paper contains a report of work on sound absorption where large areas of absorbent material are installed. The measurements show that under these conditions it is impossible to obtain a logarithmic decay, hence the sound absorption is less than would be expected from the absorption coefficient of the material.

Measurements were also made on very small areas. In this case the absorption was more than would be expected from the coefficient determined by measuring the absorption of an area of 72 square feet.

\section{CONTENTS}

II. Effect of a large amount of absorption on one surface

1. Experimental results $\ldots \ldots$

(a) Measurements with a nondirectional microphone using

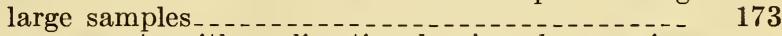

(b) Measurements with a directional microphone using large samples.............

2. Practical application

III. Effect of small areas

1. Experimental results........ 181

IV. Conclusion

2. Practical application........ 185

\section{INTRODUCTION}

The problem of measuring the sound absorption characteristics of materials and then making use of these measurements to compute the amount of material to be used in treating a room acoustically so as to obtain definite results is more complicated than was originally anticipated. When this problem was attacked by Wallace Sabine some 40 years ago, most of the rooms which he examined were fairly reverberant and the sound absorbing materials had low coefficients; but during the past few years there have been developed materials with much higher coefficients of absorption and there is a growing demand for acoustically dead rooms. As a result, it has been found 
that some of the simplifying assumptions which were made and were approximately true for the older conditions are not sufficiently accurate for the treatments of modern problems in architectural acoustics, and doubts have arisen concerning the validity of both the laboratory methods of determining absorption coefficients and the manner in which the test results should be used in the acoustical treatment of auditoriums. The results of some experiments, which have recently been carried out at the National Bureau of Standards and are described in the present paper, throw some light on these difficulties.

The acoustic qualities of a room in a large measure depend upon the rate at which sound energy in it decays. After a room is completed this rate of decay can be measured, but the problem which must be solved, is, how to design a room so one is sure what its acoustic qualities will be after it is built.

The rate of decay of sound energy has been measured in many different kinds of rooms which were used for many different purposes. In general if the room is properly designed and the absorbent material properly placed so as to prevent echoes, flutters, etc., and if it has a given rate of decay, it can be predicted whether or not it will be acoustically satisfactory for a given use, as for example, an auditorium or a business office.

To know in advance of construction that a room will be acoustically satisfactory requires, therefore, that we know how to compute this rate of decay from the geometrical dimensions of the room and the acoustical properties of the materials used in its walls, ceiling, rnd floor, and in addition the acoustical properties of the furniture and other contents of the room. In attempting to solve this problem it is customary to make acoustical measurements of various materials, furniture, etc., in a reverberation chamber, and then, using a formula to compute certain numerical coefficients for the materials, furniture, etc., which will aid us in making the necessary prediction.

The formula ${ }^{1}$ most commonly used, for surfacing materials, is the "Sabine formula" $T=\frac{.05 V}{a_{1} s_{1}+a_{2} s_{2}+\ldots}$ where $T$ is the reverberation time (in seconds); i.e., the time required for the sound to decay to $\frac{1}{1,000,000}$ of its original value, $V$ the volume (in cubic feet) of the room (or sound chamber), $s_{1}, s_{2} \ldots$.. the areas of the different surfaces (in square feet), and $a_{1}, a_{2} \ldots$ are called the sound absorption coefficients of these surfaces and $a_{1} s_{1}+a_{2} s_{2} \ldots$ is called the total absorption of the room (or chamber). Although in practice the process is simplified by a "calibration" of the chamber, in theory, the determination of the "absorption coefficient" of a surfacing material would consist in measuring the rate of decay of sound in the reverberation chamber with and without the presence of a known area of the material. From the two reverberation times so measured and the known dimensions of the chamber, the absorption coefficient would then be determined by substituting in the formula.

1 This formula has been most generally used for computation purposes in the past. Other formulas have been derived by C. E. Eyring (Reverberation Time in Dead Rooms. The Journal of the A coustical Society of America, vol. I, Jan. 1930), G. Millington (The Journal of the Acoustical Society of America, vol. IV, July 1932), and W. J. Sette (The Journal of the Acoustical Society of America, vol. IV, Jan. 1933). The ollowing discussion applies to all of these formulas. 
To determine what effect a combination of materials would have in a room, the absorption coefficients so determined are substituted in the same formula using the volume of the room and the respective areas as given by the design. The reverberation time computed in this way is expected to give some idea of the reverberation time which the room would have after it is constructed.

In deriving this formula certain assumptions are made as follows:

(1) The reflected sound energy flux over any portion of the surface is a definite fraction $(1-a)$ of the incident sound energy flux, where $a$ is defined as the absorption coefficient of the material.

(2) The sound absorption coefficient of a material is independent of the intensity of the sound.

(3) The sound energy flux incident upon the boundary surfaces of the air space enclosed in the room or chamber is uniform over all the boundary surfaces, or in other words there is a diffuse distribution of sound energy.

From these assumptions it follows:

(a) The decay of sound energy in an enclosure will be logarithmic.

(b) The absorption of a given material will be proportional to its area.

Up to the present time for the materials used, there is no experimental evidence to indicate that the second assumption is not correct. When the absorption of a material is small the other two assumptions are approximately correct, but as the absorption becomes larger the third assumption ceases to be even a rough approximation in most cases. The condition assumed under (3) can be partly controlled by the experimenter, but it has been found that even in a reverberation chamber, where the source is rotating and rotating vanes are installed, it is impossible, when the sample is highly absorbent, to meet exactly this third condition.

As a result of this inability to meet condition (3), when measurements are made in a reverberation chamber, the coefficient which is computed, in general, cannot represent the coefficient as defined by assumption (1) upon which the formula was based; that is, the fractional part of the incident energy which is absorbed at a single reflection. Furthermore, absorption coefficients of highly absorbent materials determined by any method which locates only two points on the decay curve, are inadequate for purposes of design.

\section{EFFECT OF A LARGE AMOUNT OF ABSORPTION ON ONE SURFACE}

\section{EXPERIMENTAL RESULTS}

We will now proceed to discuss the results of certain recent experiments ${ }^{2}$ which have been carried out in the reverberation chamber at the National Bureau of Standards. As the dimensions of this room probably affect the results to some extent, it is desirable to restate them. The floor is approximately $25 \mathrm{ft}$. by $30 \mathrm{ft}$. and the ceiling height is $20 \mathrm{ft}$. In these reverberation chamber measurements, every possible attempt has been made to fulfill the condition (3) assumed in the derivation of the formula. Curve 1 in figure 1 shows that in

${ }^{2}$ A description of the equipment used will be found in BS Research Paper RP459, "An Automatic Reverberation Meter for the Measurement of Sound Absorption." 
the empty room a logarithmic decay is obtained within experimental error. It was also found that, when a sample of $72 \mathrm{sq} f \mathrm{ft}$ having a small coefficient of absorption was placed in the chamber, the decay of sound energy in the chamber was still logarithmic within experimental error but, when a highly absorbent sample of $72 \mathrm{sq} f t$ was used the decay was no longer logarithmic as shown in curve 2 of figure 1.

As this decay was not logarithmic it was assumed there was not a diffuse distribution of sound energy, hence it was decided to install rotating vanes. ${ }^{3}$ Figure 2 shows the interior of the reverberation chamber and gives some idea of the relative size of the vanes and chamber. The loud speakers are mounted in the vanes which serve as baffles. Also, the speakers rotate with the vanes and these moving sources tend to give a more uniform distribution of the sound energy.

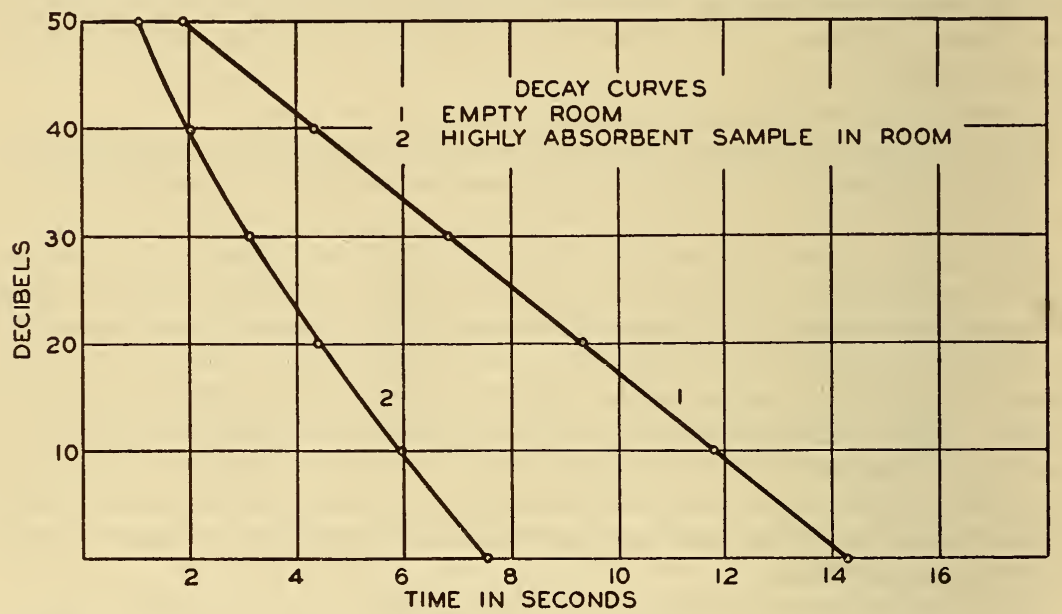

FIGURE 1.-Curves showing logarithmic decay in empty room and a nonlogarithmic decay when a highly absorbent sample is in the chamber

It should also be noticed that these vanes are tipped at an angle of approximately $15^{\circ}$ from a vertical position. This tends to change sound waves which are traveling in a vertical direction to a horizontal direction and those traveling in a horizontal direction to a vertical. As the vanes rotate this also rotates the sound field about a vertical axis. As a result of these two actions it might be said that the sound field is being rotated about two axes perpendicular to each other, hence a better distribution of sound energy should be obtained.

Figure 3 shows the results obtained after the vanes were installed when a highly absorbent sample of $72 \mathrm{sq} \mathrm{ft}$ was again placed in the reverberation chamber. Under these conditions a logarithmic decay of the sound energy was obtained within the experimental error of measurement. As this decay was logarithmic it was assumed that a fairly diffuse distribution of sound energy was obtained by means of the rotating sources and rotating vanes. All of the results given in the rest of the paper are from measurements taken when the vanes were rotating.

3 The Measurement of Sound Absorption Coefficients by Paul E. Sabine, Journal of the Franklin Institute, 207(1929). 


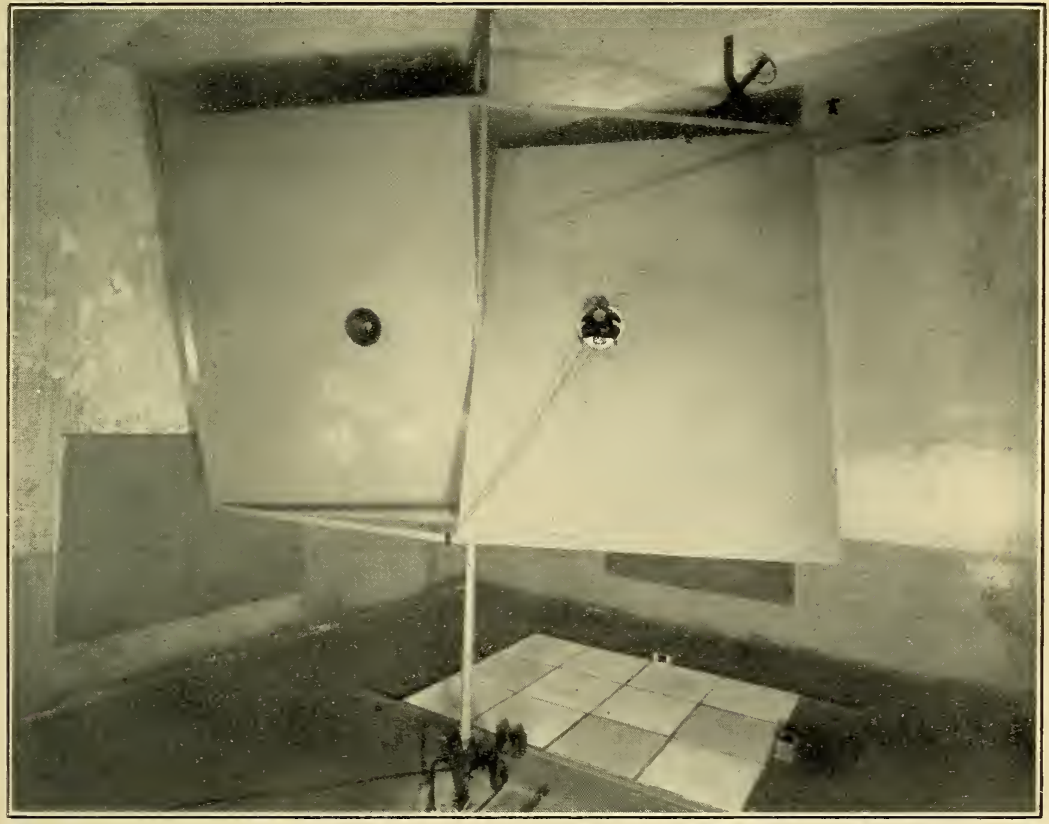

Figure 2.-Interior of reverberation chamber at National Bureau of Standards. 
(a) MEASUREMENTS WITH A NONDIRECTIONAL MICROPHONE USING LARGE SAMPLES

It has been known for some time that a satisfactory acoustical treatment cannot be obtained by putting a highly absorbent material only on the ceiling, especially if the ceiling is fairly high. The sound which strikes the highly absorbent material is quickly absorbed but that which strikes the untreated parallel walls is reflected back and forth between these walls and lasts for an appreciably longer time than that between the floor and ceiling. In order to study the decay curves in such a room, enough of each of 3 different kinds of material was obtained to cover the entire floor of the reverberation chamber. The coefficients of these samples are given in table 1. Measurements were made on sample no. 1 at 128, 256, 512, 1,024, 2,048, and 4,096

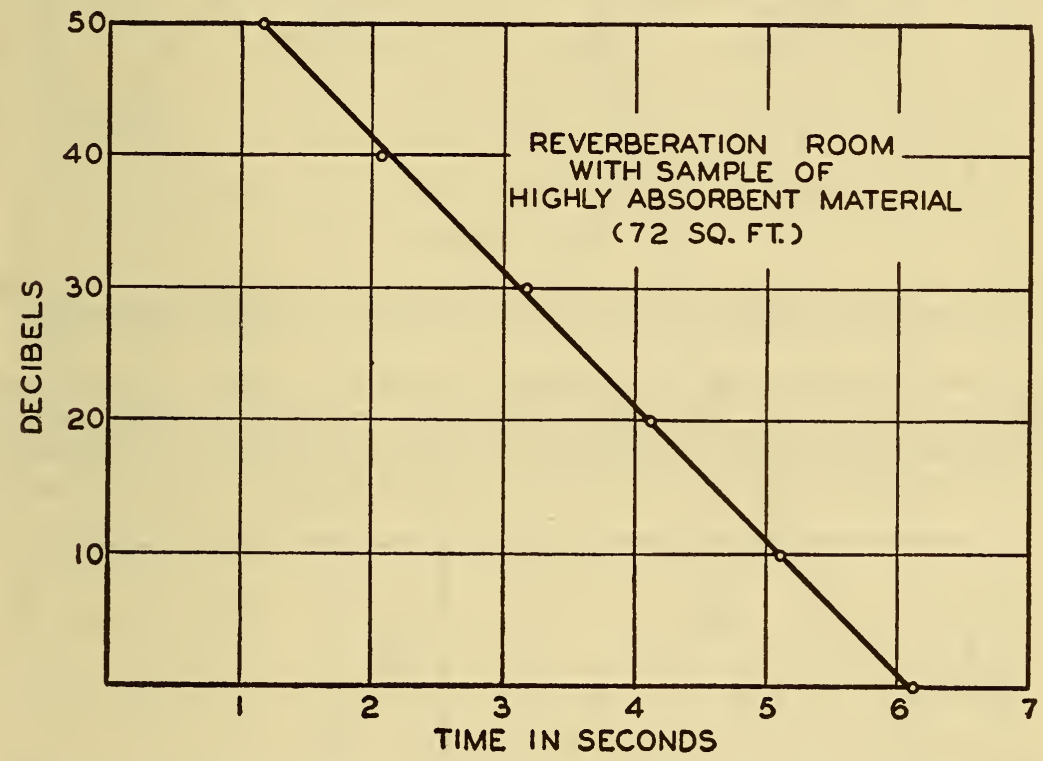

FIGURE 3.-Curve showing that a logarithmic decay is obtained, when a highly absorbent sample is in the chamber, after rotating vanes are installed

cycles when approximately one-fourth, one-half, and the whole of the floor was covered. Figure 4 shows the form of decay curves obtained at 512 cycles. In all three cases the decay of sound energy has ceased to be logarithmic, hence it is assumed that there is not a diffuse distribution of sound energy.

The straight lines which are drawn approximately tangent to the upper portions of the curves represent the way the sound should have decayed as computed by the formula. For these computations the coefficient .83 was used, which was the value determined by measurements on a $72 \mathrm{sq} \mathrm{ft}$ sample. It should be noticed that the sound starts to decay at the computed rate but the rate becomes smaller and smaller with increased time. The divergence of the measured time from the computed time, which was determined by using the Sabine formula and the coefficient given above, is not greatly different for the three areas. It is important, however, to notice that after a decay of 60 decibels the relative increase in time as measured by the 
curve over that indicated by the straight line computed time is quite small for the case where one-quarter of the floor is covered, but when the entire floor is covered this relative increase is approximately 100 percent.
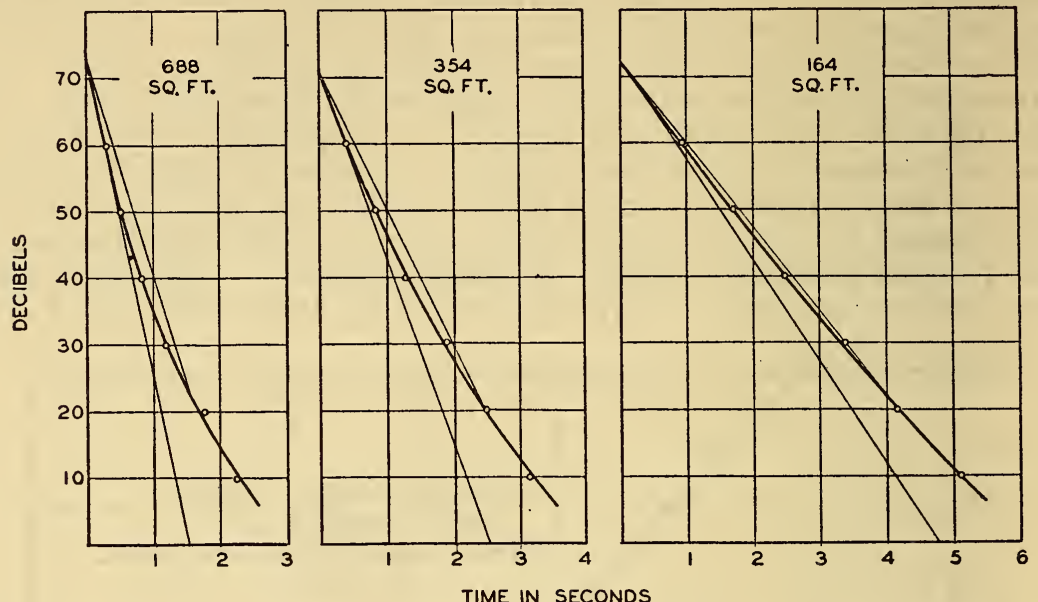

FIgURE 4.-Decay curves at 512 cycles when approximately all, $1 / 2$ and $1 / 4$ of the floor of the reverberation chamber was covered with sample no. 1 (dynamic microphone)

The next question was what would happen if a material with a low coefficient were used. To determine this, a material (sample no. 2) was chosen which had a coefficient of .19 at 512 cycles. Figures 5 and 6 show the decay curves, the whole floor being covered. It is to

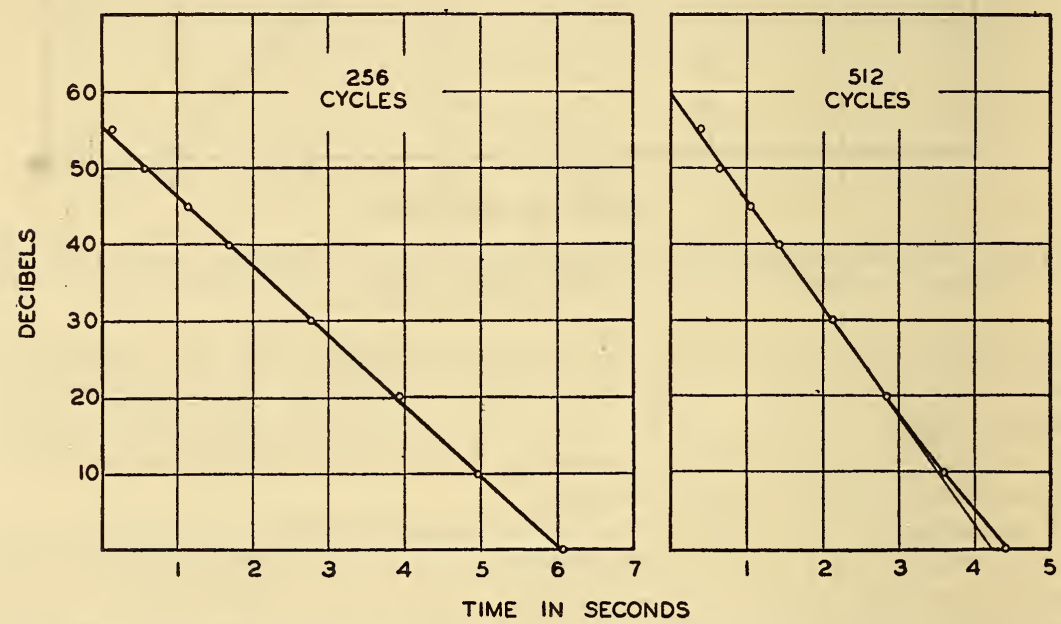

FIGURE 5.-Decay curves at 256 and 512 cycles when the floor of the reverberation chamber was covered with sample no. 2 (dynamic microphone)

be noticed that at 256 cycles the decay is logarithmic through the range over which we were able to take measurements. In this case the coefficient of absorption is small, (see table 1) the total absorption being only 79 units. At 512 cycles where the absorption becomes 
larger, 137 units, the decay is no longer logarithmic, while at 1,024 and 2,048 cycles, where the absorption is still larger, the divergence from a logarithmic decay is still greater. Figure 7 shows the decay curves at 256 and 512 cycles for sample no. 3 , which at these frequencies had absorption coefficients (see table 1) intermediate between these of samples nos. 1 and 2.

From the measurements on these samples it appears that the divergence of the measured rate of decay, caused by placing absorbent material on one surface of a room, is primarily a function of the total amount of absorption and within the error of our measurements was
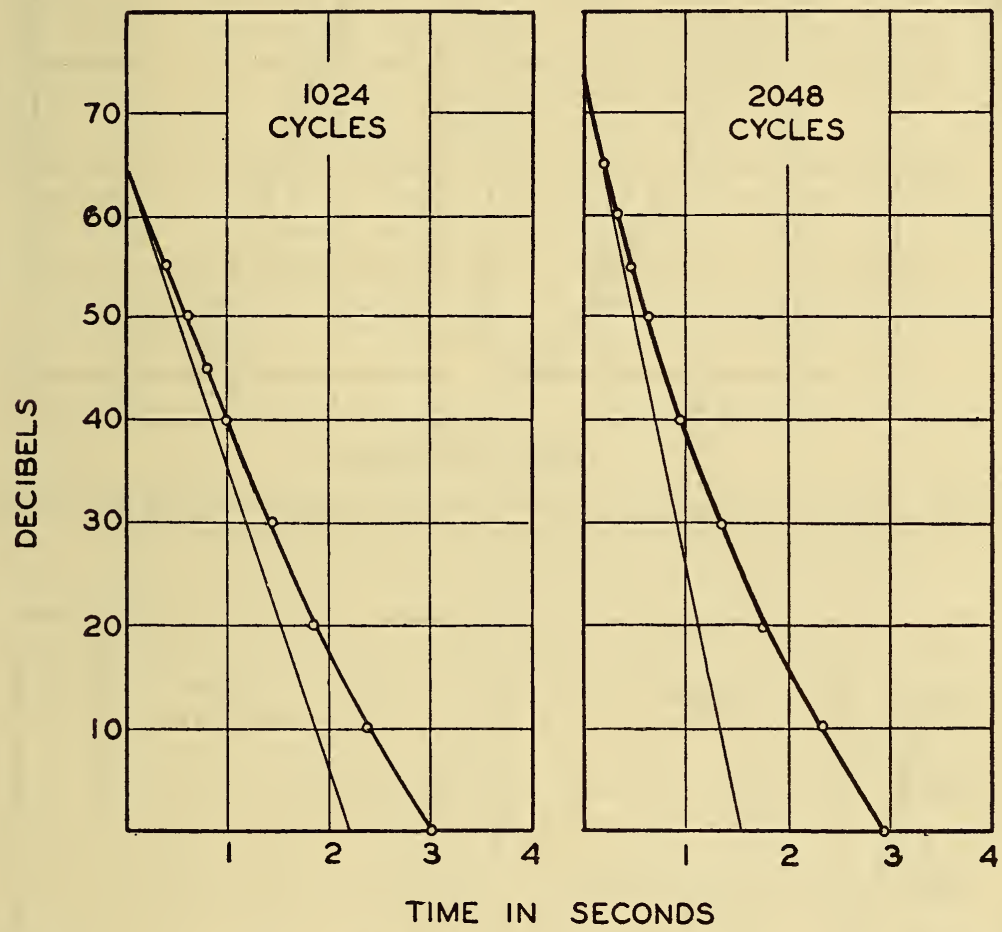

FIGURE 6.-Decay curves at 1,024 and 2,048 cycles when the floor of the reverberation chamber was covered with sample no. $\mathbb{Z}$ (dynamic microphone)

independent of the frequency, for frequencies under 2,500 cycles. From these measurements and a large number of others it has been found that when approximately 110 units of absorption are placed on the floor of this reverberation chamber which has an area of 750 sq $\mathrm{ft}$, the sound decay deviates measurably from logarithmic decay, even when a rotating source and rotating vanes are used. In other words, when the total absorption placed on the floor is numerically equal to about one-seventh the area of the floor, a nonlogarithmic decay is obtained. To obtain some idea as to whether this relation was approximately the same in enclosures of a different size similar measurements were made in a $6-\mathrm{ft}$ steel box which was cubical in shape. It was found that about the same ratio between the total absorption and area of surface held as in the reverberation chamber. 


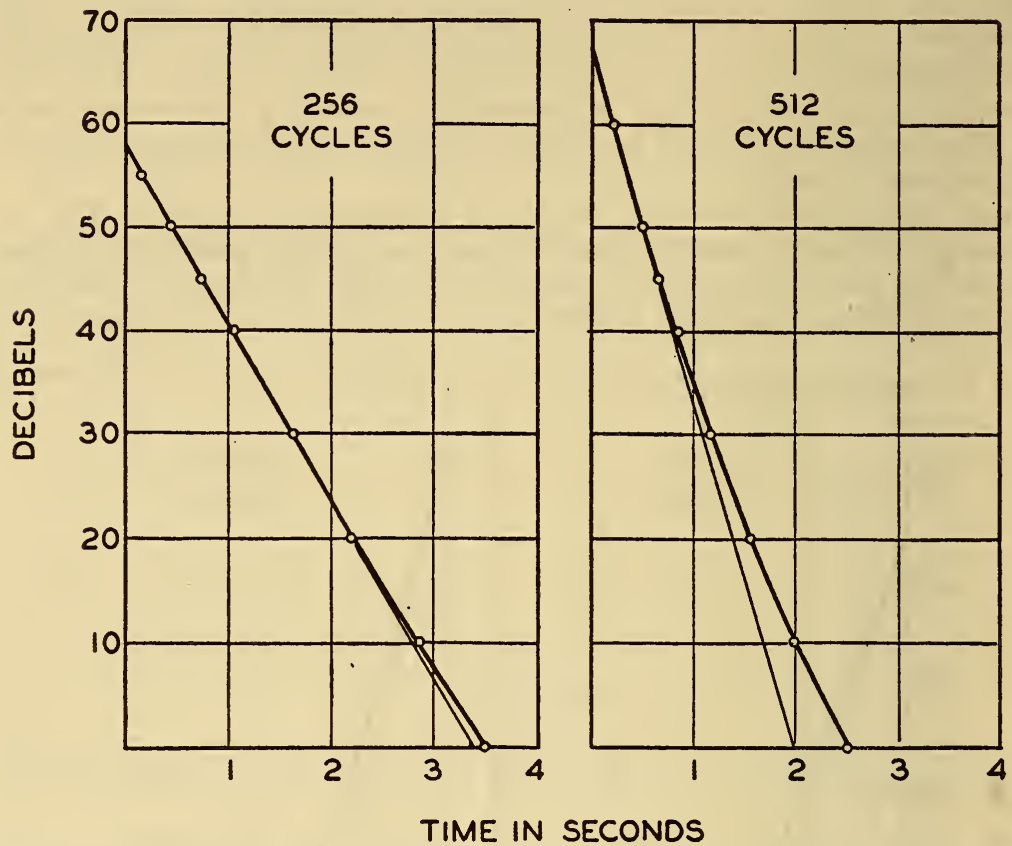

Figure 7.-Decay curves at 256 and 512 cycles when the floor of the reverberation chamber was covered with sample no. 3 (dynamic microphone)
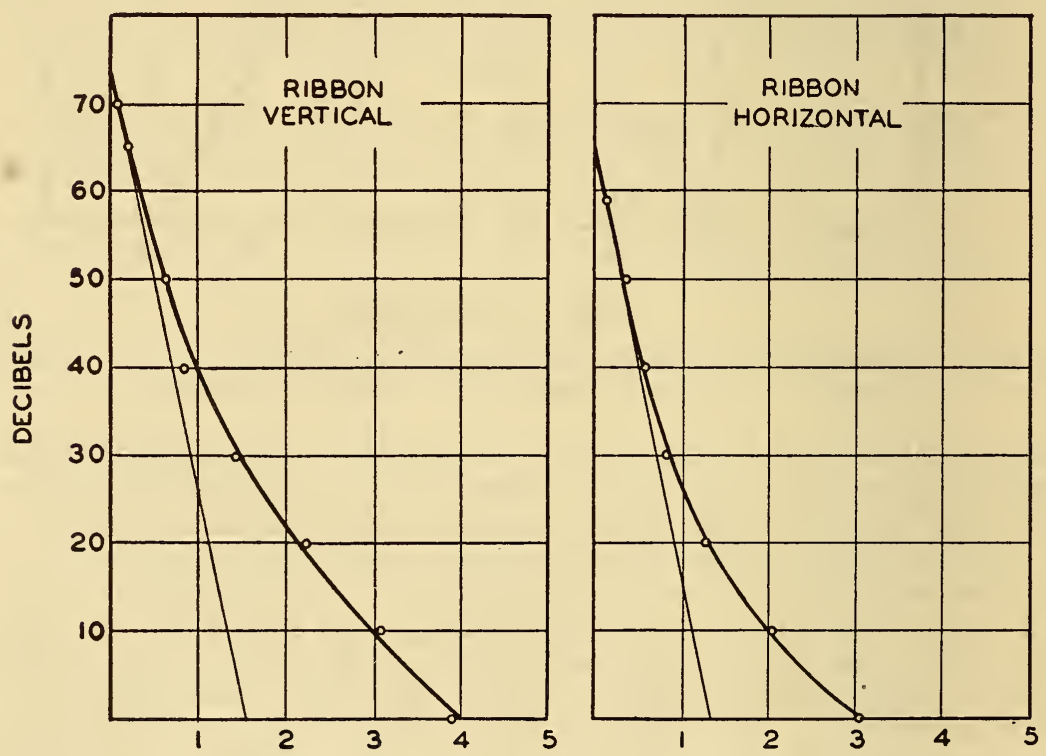

TIME IN SECONDS

FIGURE 8.-Decay curves at 512 cycles when the floor of the reverberation chamber was covered with sample no. 1 (ribbon microphone) 
These results might be summarized as follows: When a very small amount of absorption is placed on the floor of the reverberation chamber the decay of sound energy is logarithmic. If this absorption is increased, the decay is still approximately logarithmic for a short time after the sound is cut off, but it becomes less and less so with time. When the entire floor is covered with a highly absorbent material, the decay is not even approximately logarithmic an instant after the loud-speaker is cut off.

(b) MEASUREMENTS WITH A DIRECTIONAL MICROPHONE USING LARGE SAMPLES

All of the measurements given up to the present time were taken with either a condenser or dynamic microphone. Both of these microphones are essentially nondirectional in character. To obtain further evidence as to the distribution of the sound energy it was decided to take some measurements with a microphone, such as a ribbon microphone, which had directional characteristics. Figure 8 shows the results of some of these measurements with practically the whole floor covered with sample no. 1 . It will be noticed that the general slopes of the curves are very similar to those taken with the dynamic microphone. There are two important differences in these curves which should be noted. When the ribbon was vertical, the microphone was measuring primarily the sound reflected between the untreated walls. When the ribbon was horizontal, the microphone was measuring primarily the sound reflected between the floor and the ceiling. It will be noticed that the sound level, as indicated by the intersections of these curves with the vertical axis, was higher between the untreated walls than it was between the floor and ceiling, and that the rate of decay was not exactly the same for the two cases. In other words, the measurements show that the distribution of sound flux was markedly different in different directions and that, as in the case of the measurements with the dynamic microphone, the decay is not even nearly logarithmic an instant after the speakers were cut off. Figure 9 shows the results for sample no. 2 at 512 cycles. For this sample, the sound level for 512 cycles was the same in the two directions within the error of measurement at the time the speaker was cut off. Judging from the decay curves a logarithmic decay was maintained approximately for the first 50 or $55 \mathrm{db}$ of the decay, but as the decay continued it became nonlogarithmic.

In the case of the $72 \mathrm{sq} \mathrm{ft} \mathrm{sample} \mathrm{these} \mathrm{differences} \mathrm{have} \mathrm{not} \mathrm{been}$ found to exist. The sound level is the same in the two directions and there is a logarithmic decay as with the dynamic microphone.

Table 1 gives the coefficients for the samples as computed from the measurements on an area of $72 \mathrm{sq} \mathrm{ft}$. In addition "absorption coefficients" are given computed from the decay curves obtained when $1 / 4$, $1 / 2$, and the whole of the floor was covered. A chord (see fig. 4) was drawn across the first 50 decibels of the decay curve and the "absorption coefficient" computed on the assumption that the decay had followed this logarithmic curve. 
TABLE 1

SAMPLE NO. 1: DYNAMIC MICROPHONE

\begin{tabular}{|c|c|c|c|c|c|c|}
\hline \multirow{2}{*}{ Area of material } & \multicolumn{6}{|c|}{ Sound-absorption coefficients for frequencies } \\
\hline & 128 & 256 & 512 & $1024 \overline{3}$ & 2048 & 4096 \\
\hline $\begin{array}{l}72 \text { square feet area } \\
14 \text { floor covered (chord) } \\
1 / 2 \text { floor covered (chord) } \\
\text { Entire floor covered (chord) }\end{array}$ & $\begin{array}{r}0.46 \\
.42 \\
\end{array}$ & $\begin{array}{r}0.76 \\
.68 \\
.63 \\
.54\end{array}$ & $\begin{array}{l}0.83 \\
.67 \\
.59 \\
.58\end{array}$ & $\begin{array}{r}0.82 \\
.69 \\
.54 \\
.49\end{array}$ & $\begin{array}{r}0.81 \\
.77 \\
.64 \\
.47\end{array}$ & $\begin{array}{r}0.78 \\
.75 \\
.64 \\
.47\end{array}$ \\
\hline
\end{tabular}

SAMPLE NO. 1: RIBBON MICROPHONE 5 FEET ABOVE FLOOR

\begin{tabular}{|c|c|c|c|c|c|}
\hline $\begin{array}{l}72 \text { square feet area: } \\
\text { Ribbon vertical } \\
\text { Ribbon horizontal } \\
\text { Entire floor covered: } \\
\text { Ribbon vertical (chord) } \\
\text { Ribbon horizontal (chord) }\end{array}$ & $\begin{array}{r}0.56 \\
.55\end{array}$ & $\begin{array}{r}0.81 \\
.82 \\
.43 \\
.49\end{array}$ & $\begin{array}{r}0.87 \\
.85 \\
.54 \\
.70\end{array}$ & $\begin{array}{r}0.51 \\
.60\end{array}$ & 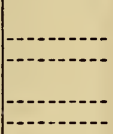 \\
\hline
\end{tabular}

SAMPLE NO. 2: DYNAMIC MICROPHONE

\begin{tabular}{l}
\hline 72 square feet area \\
Entire floor covered (chord)
\end{tabular}

SAMPLE NO. 2: RIBBON MICROPHONE 5 FEET ABOVE FLOOR

Entire floor covered:
$\quad$ Ribbon vertical (chord)
$\quad$ Ribbon horizontal (chord)

SAMPLE NO. 3: DYNAMIC MICROPHONE

72 square feet area
Entire floor covered (chord)

The foregoing results may partially explain the difference sometimes found between the sound-absorption coefficients obtained by the ear method, which as usually carried out, determines only two points on the decay curve, and those obtained by determining several points on the curve. It is possible that the decay, even under the best conditions, when a highly absorbent sample of $72 \mathrm{sq} \mathrm{ft}$ is present in this reverberation chamber, is not logarithmic down to the threshold of the ear, although it is logarithmic within experimental error for the first $70 \mathrm{db}$. We hope to make further improvements in our equipment so that it will be possible to make measurements at these lower sound intensities which will settle this point.

\section{PRACTICAL APPLICATION}

It is also desired to call attention to the fact that these curves may aid one in making a better estimate as to what may be expected where there is an acoustical treatment which is applied only on the ceiling. To illustrate this, let us take as an example a room, on which we have measurements, which was $103 \frac{1}{2} \mathrm{ft}$ long, $23 \mathrm{ft}$ wide, and $13 \mathrm{ft}$ high. In this case all of the acoustical treatment was on the ceiling. Figure 10 shows the decay curves as taken with our measuring equipment using a nondirectional microphone. 
Using the formula $T=\frac{.05 \mathrm{~V}}{A S}$ and the coefficients of the different surfaces in the room, the computed reverberation time came out 1.29

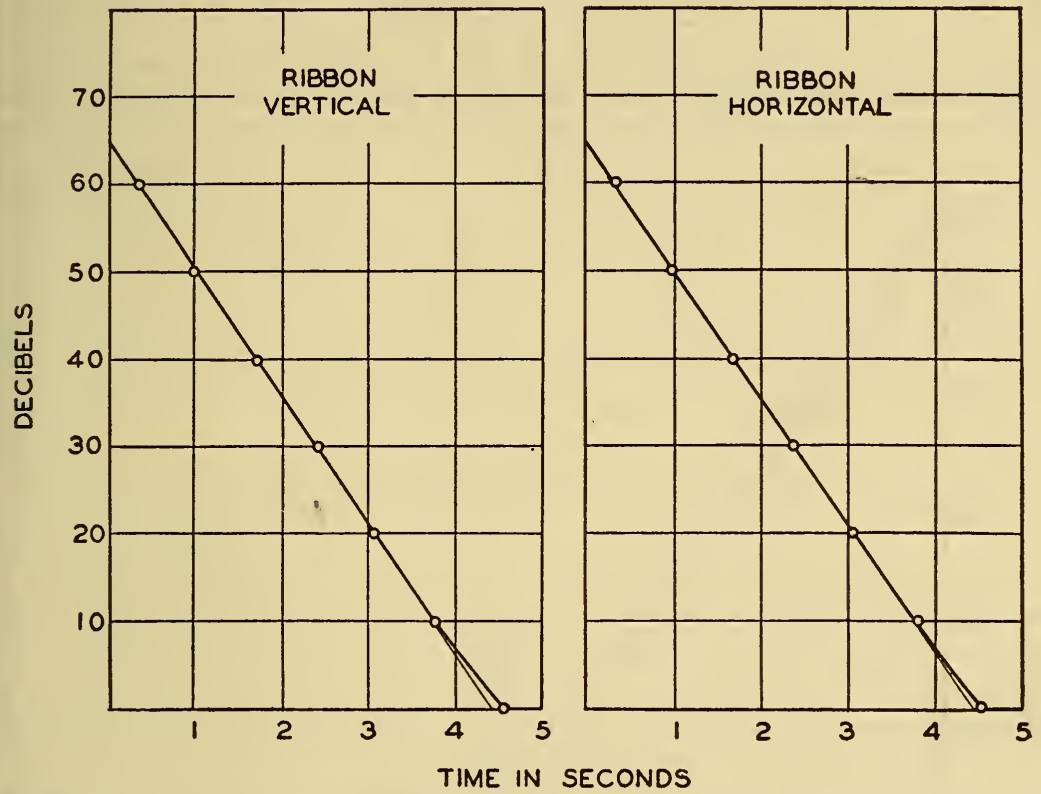

FIGURE 9.-Decay curves at 512 cycles when the floor of the reverberation chamber was covered with sample no: 2. (ribbon microphone)

seconds at 512 cycles. This is less than the measured time (1.46 seconds) shown in figure 10 . If we make use of the results given in figure 7 , in which the sample had approximately the coefficient of

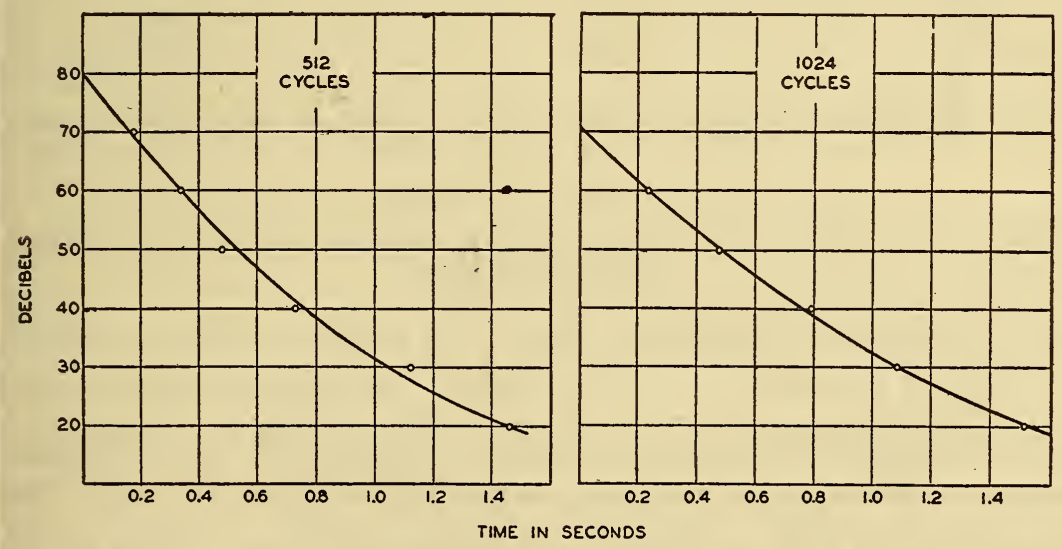

FIGURE 10.-Decay curves in large room having a ceiling treatment

the material used as the ceiling treatment in this room, we should be able to estimate approximately the time for a $60 \mathrm{db}$ decay. Referring to figure 7 we find that the time required for a $60 \mathrm{db}$ decay for the 
sample was 2.1 seconds at 512 cycles, while if it had decayed logarithmically it should have required only 1.8 seconds. Assuming a similar divergence from a logarithmic decay in the room mentioned above, the time should be $1.29 \times \frac{2.1}{1.8}=1.5$ seconds. This is approximately the time measured as shown by figure 10. Measurements were also made by the ear method with an organ pipe and stop watch. The initial sound level in this case was $76 \mathrm{db}$ above the threshold of the

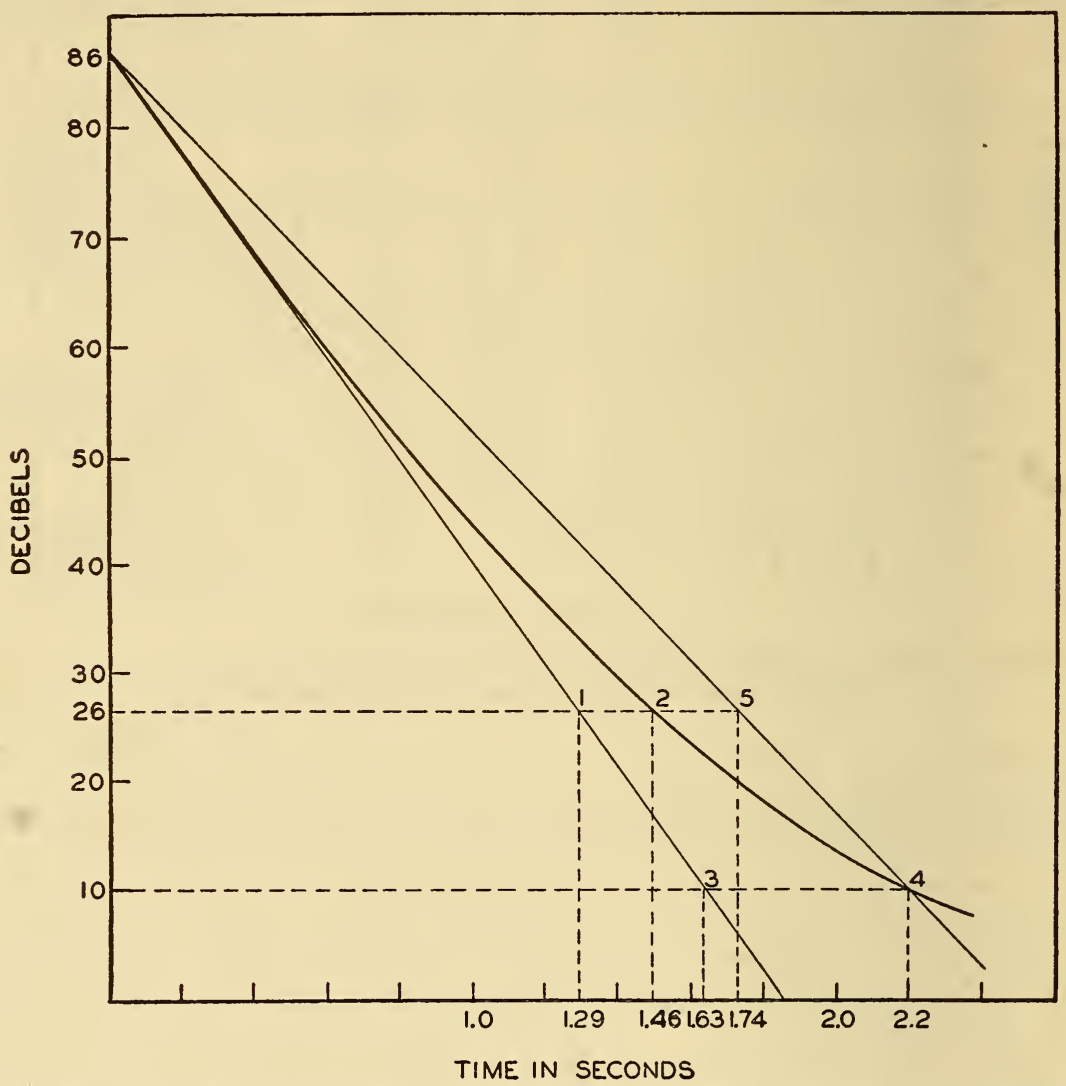

FIGURE 11.--Graphical illustration of difference between measured and computed reverberation time

ear. Assuming a logarithmic decay the computed time would be $1.29 \times \frac{76}{60}=1.63$ seconds for a $76 \mathrm{db}$ decay. In this case it is necessary to extend the curves slightly in figure 7. Doing this and making the computation as before, the time becomes $1.63 \times \frac{3.1}{2.3}=2.2$ seconds. The time measured with the organ pipe was also $2.2+$ sec. These results check rather closely, in fact, better than should be expected. Other rooms on which we have made measurements have not checked as well as this one, but at most the error did not exceed .1 of a second. Figure 11 gives a graphical representation of these results. Point (1) 
represents the computed time for a $60 \mathrm{db}$ decay. This would also be the correct time if the decay were logarithmic. Point (2) represents the actual time of decay in this room, due to the fact that the decay was not logarithmic. In the same way, point (3) is the computed time for a $76 \mathrm{db}$ decay provided it is logarithmic, point (4) is the actual time for a $76 \mathrm{db}$ decay, and point (5) is the time for a $60 \mathrm{db}$ decay obtained by the ear method using an organ pipe which gave an intensity of $76 \mathrm{db}$ above the ear threshold. It is of interest to note that the time computed by the usual method from the measurement made with this calibrated organ pipe for a $60 \mathrm{db}$ decay is .2 of a second too long, while that computed from the formula is .2 second too small.

In general, the time for a $60 \mathrm{db}$ decay can not be computed correctly by use of the formula, neither can it be measured correctly by determining two points on the decay curve. It is hoped, however, that if enough decay curves are obtained for different types of treatment that the time for a $60 \mathrm{db}$ decay can be approximately close enough for all practical purposes, in some such manner as just indicated.

The above discussion applied primarily to small rooms of less than 30,000 cubic feet. For larger rooms it is probable the divergences are much smaller.

Another case might arise where all of the material is located on the side walls and none on the ceiling. This is entirely different and the curves shown would not apply. Attention should also be called to the fact that these curves apply only to a room which has practically no absorption on the walls. As soon as a small amount of absorbent material is placed on the walls, the decay becomes more nearly logarithmic and the measured time for a $60 \mathrm{db}$ decay approaches that which one would compute from the Sabine formula.

\section{EFFECT OF SMALL AREAS}

\section{EXPERIMENTAL RESULTS}

Another problem in measuring absorption coefficients arises from the fact that condition (b), "The absorption of a given material will be proportional to its area" is generally not met although the decay is logarithmic within experimental error. As a result of this, it becomes necessary to choose some size of sample, for reverberation chamber measurements, from which an arbitrary coefficient can be determined which can be used for design purposes.

When the measurement of sound absorption by the reverberation room method was first started at the Bureau of Standards, consideration was given to this problem, and judging from the work of Paul E. Sabine ${ }^{4}$ an area of 60 to $70 \mathrm{sq} \mathrm{ft}$ was deemed large enough to give satisfactory results. An area of $72 \mathrm{sq} \mathrm{ft}$, or in other words a sample 8 by $9 \mathrm{ft}$ was finally chosen.

During the past few years there has been a race among the various manufacturers of acoustical materials to develop a material having the highest possible absorption. As a result, materials have been developed which have a very high absorption. It was evident, when measurements were made on such materials, that the coefficients computed from measurements on areas of $72 \mathrm{sq} \mathrm{ft}$, were incorrect because in some cases they exceeded 100 percent. It then became

4 See footnote 3 . 
necessary to make a further investigation of this problem. To obtain some idea of the effect of area, absorption measurements have been made on different samples of various sizes ranging from $4 \mathrm{sq} \mathrm{ft}^{\mathrm{t}}$ to $144 \mathrm{sq} \mathrm{ft}$. For the smaller areas (4, 9, and $16 \mathrm{sq} \mathrm{ft}$ ) measurements were first made on a single area. These measurements were repeated many times to eliminate the high probable error of an individual determination caused by the small change in total absorption of the chamber. Later a number of equal small areas were used, giving a

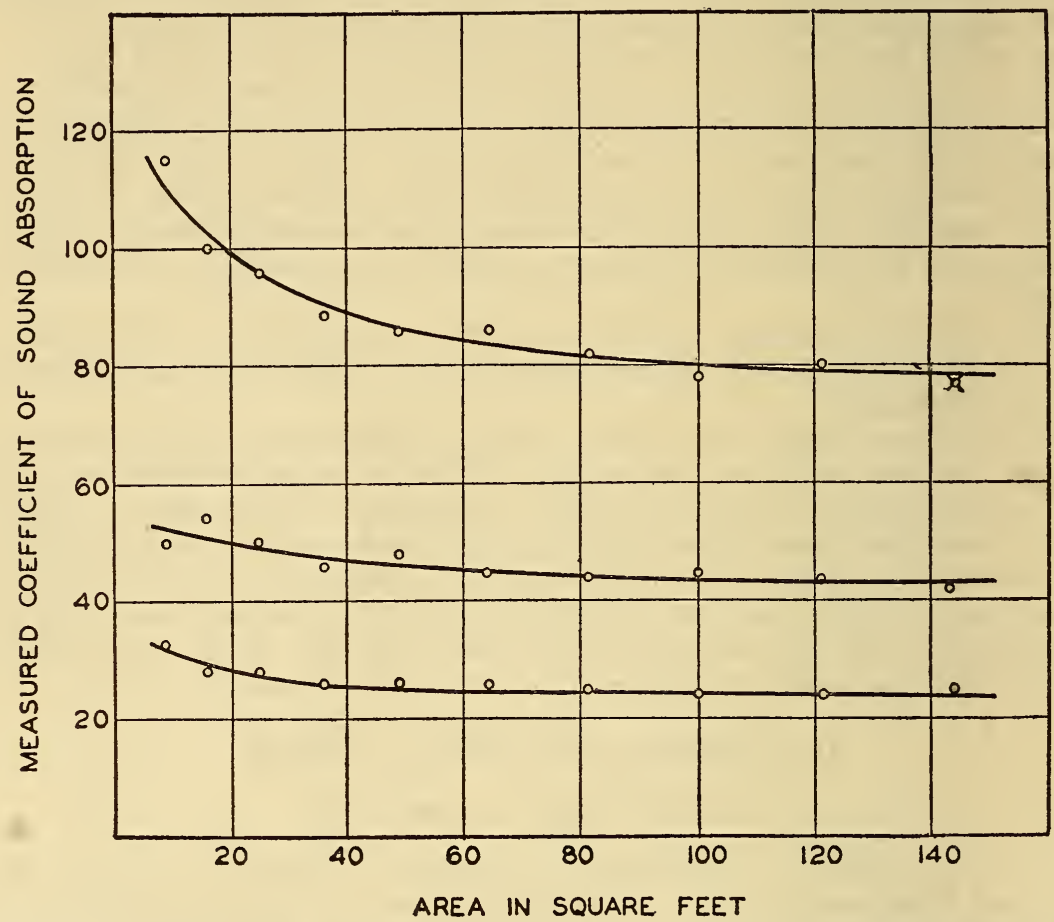

FIGURE 12.-Variation in measured coefficient with area and magnitude of absorption coefficient at 256 cycles

total area of 64 or $72 \mathrm{sq} f t$. In all these cases the areas were separated by more than the width of each (square) area.

The greater total absorption made these determinations easier and the computed absorption coefficients showed no significant difference from the results obtained with a single area. Some of the later measurements on areas of 4,9 , and $16 \mathrm{sq} \mathrm{ft}$ were therefore not made on a single area, but by the easier method of several areas. The results of all of these mesaurements on areas varying from 4 to $144 \mathrm{sq} \mathrm{ft}$ will be found in figures $12,13,14$, and 15 .

It will be noticed that when the absorption coefficient did not exceed .60, as was the case in the earlier work, the computed coefficient was constant for areas of $72 \mathrm{sq} \mathrm{ft}$ and upward so that coefficients determined on samples of this size could be used satisfactorily in computing the effect of larger areas.

For more recent samples with higher absorption, the computed coefficient still decreases with increasing area, at areas greater than 
$72 \mathrm{sq} \mathrm{ft}$, which would suggest that more satisfactory values of the coefficient would be obtained by using still larger areas. This would be the case were it not for the appearance of nonlogarithmic decay with the larger areas. Figure 16 shows the decay curve for a highly absorbent (coefficient .95) sample of $144 \mathrm{sq} \mathrm{ft}$. The two lower points lie definitely to the right of the straight line. This was the case for all $144 \mathrm{sq}$ ft samples whose computed coefficient was greater than .80. In computing the coefficients in these cases, the slope as indicated by the first four points was used and the last two points ignored. The points marked $\mathrm{X}$ on the curves shown in figures 12,

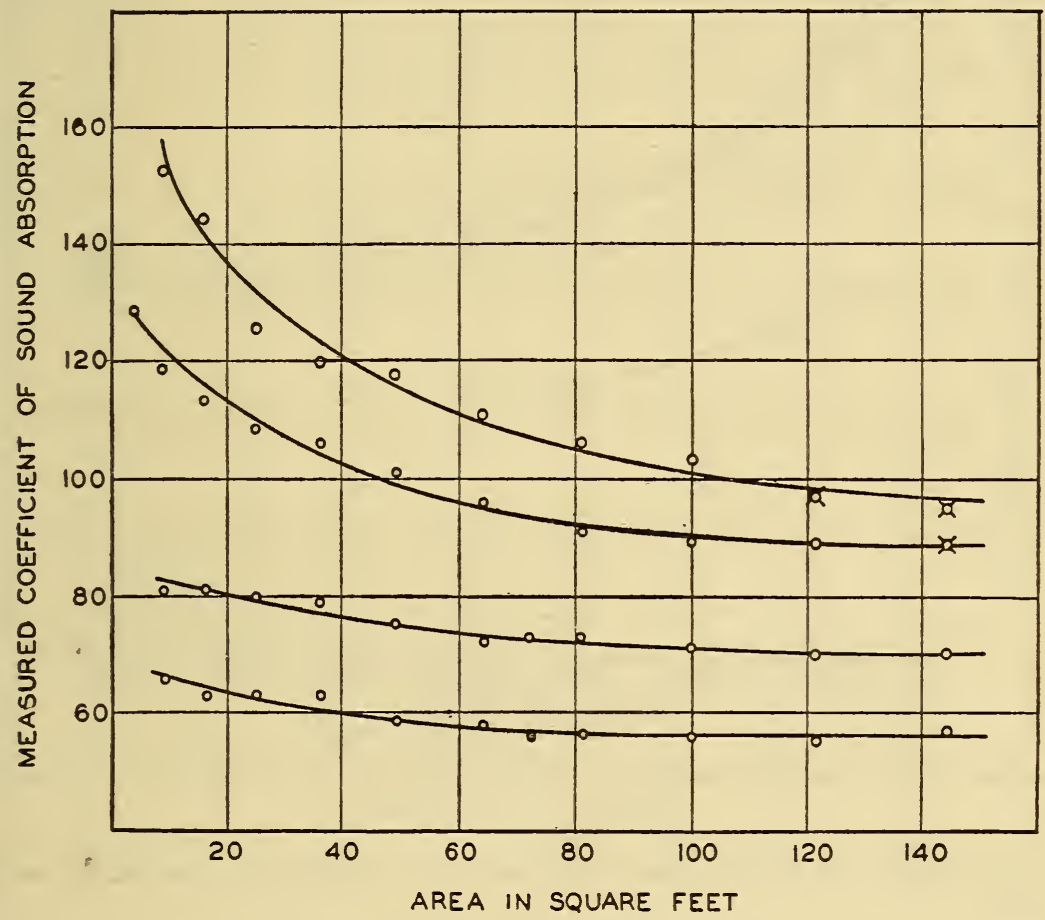

FIGURE 13.-Variation in measured coeffcient with area and magnitude of absorption coefficient at 512 cycles

13, and 14 were computed from decay curves which showed slight signs of nonlogarithmic decay. In most cases the last point was the only one which deviated from the straight line by an amount greater than the error of measurement. This complication of nonlogarithmic decay as well as the added expense of larger samples made it seem desirable to continue the use of the $72 \mathrm{sq} \mathrm{ft}$ area and take account of the affect of area by a correction term.

Examination of the curves of figures 12, 13, 14, and 15 shows that within practical limits, the shape of the curves is independent of the frequency, depending only on the relative absorption. All of the curves are nearly horizontal for areas greater than $120 \mathrm{sq} \mathrm{ft}$, so it seemed reasonable to assume that for sufficiently large areas, providing there was a diffuse distribution of sound energy, the computed coefficients would, even for material with a high absorption, be inde- 
pendent of the area. For these cases the curves are sufficiently definite to allow, by extrapolation, a reasonable determination of this limiting value of the absorption coefficient.

In this manner a table of corrections, table 2, has been obtained by which measurements made in the National Bureau of Standards

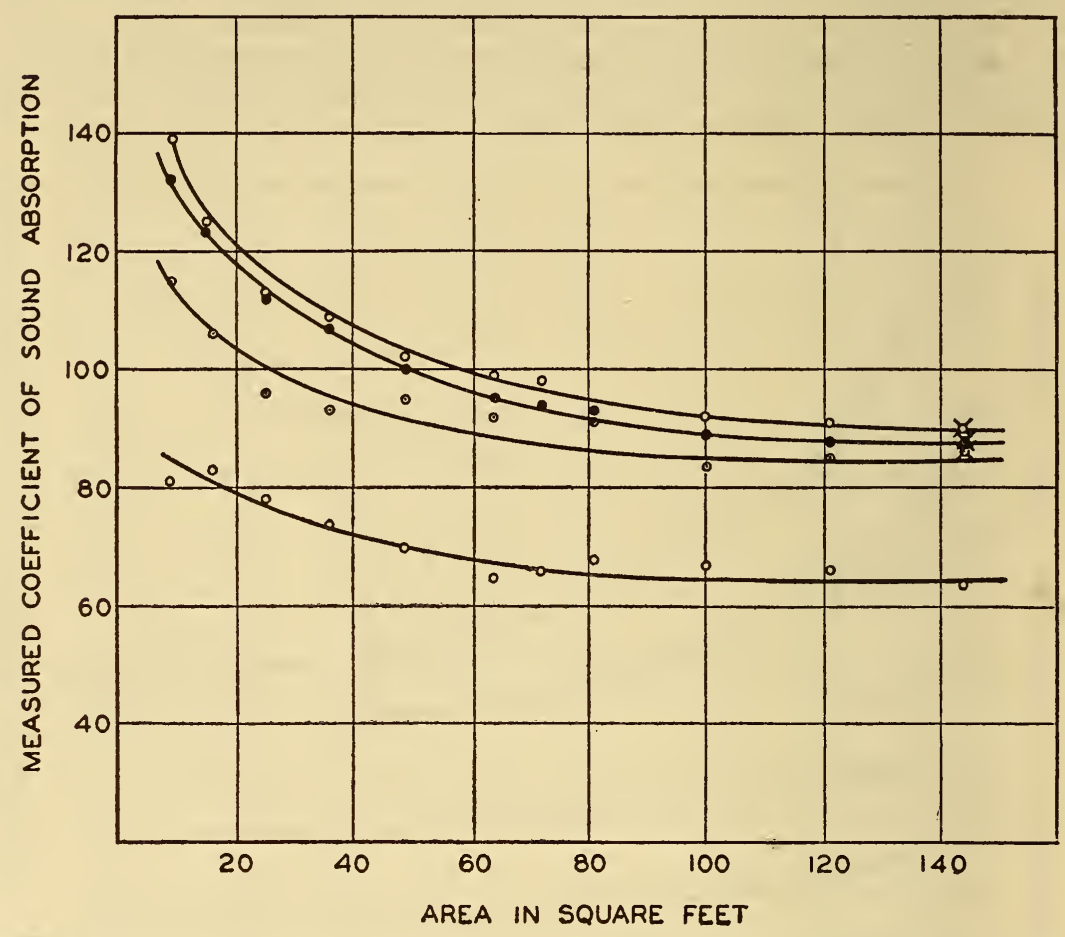

FIGURE 14.-Variation in measured coefficient with area and magnitude of absorption coefficient at 1,024 cycles

on samples of $72 \mathrm{sq} \mathrm{ft}$ are corrected to the limiting value for large areas.

TABLE 2.-Correction factors to be subtracted when absorption measurements are made on a sample having an area of 72 square feet

\begin{tabular}{|c|c|c|c|}
\hline Measured value for $72 \mathrm{sq} \mathrm{ft}$ & Correction & Measured value for $72 \mathrm{sq} \mathrm{ft}$ & Correction \\
\hline $\begin{array}{l}1.05 \\
0.95 \\
0.90\end{array}$ & $\begin{array}{l}0.09 \\
.07 \\
.06 \\
.05 \\
.05\end{array}$ & 0.80 & $\begin{array}{r}0.04 \\
.03 \\
.02 \\
.01 \\
.00\end{array}$ \\
\hline
\end{tabular}

There is reason to believe that the above corrections are independent of the room. The basis for this is that we have made a few measurements on a $4 \mathrm{sq} \mathrm{ft}$ sample in a 6 - $\mathrm{ft}$ steel cubical box and find that we obtain the same values as when the measurements are made in the reverberation chamber on the same size of sample. 
As far as we know, no one has given a satisfactory explanation why these high absorption values are obtained. It was first suggested that they were due to the absorption of the edges, but in all of the above measurements the edges were covered with angle iron. In view of this fact, the absorption of the edges was very small and this increased absorption must be due to diffraction or some phenomenon of a similar nature.

\section{PRACTICAL APPLICATION}

There is also a rather important practical application of the results shown in figures $12,13,14$, and 15 . When material is in-

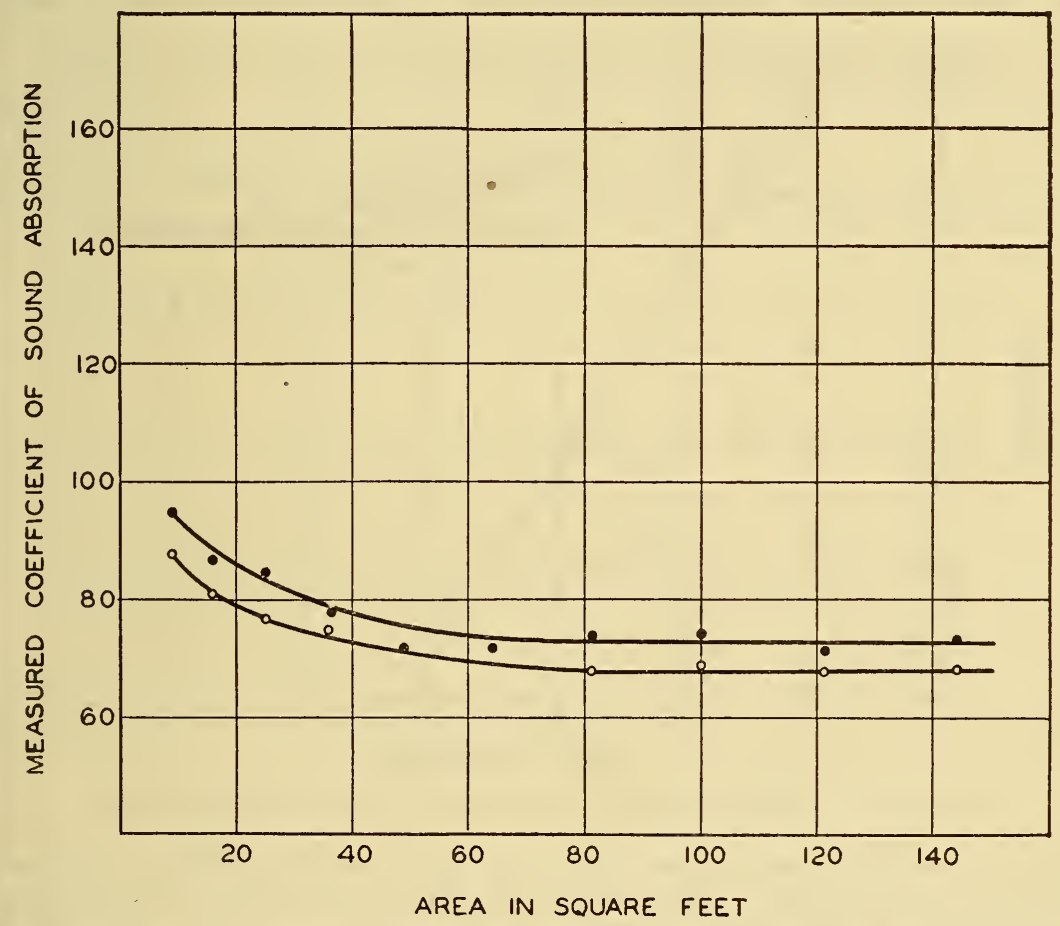

FIGURE 15.-Variation in measured coefficient with area and magnitude of absorption coefficient at 2,048 cycles

stalled in small panels the absorption will be greater than indicated by the coefficient. Unless the distance between the panels is sufficient, the increased absorption shown in the above curves will not be obtained. If the distance is too small, the absorption is less and as this distance is further decreased, the absorption is also decreased until finally the absorption becomes that which would be expected from the coefficient when the panels become one continuous area.

We have tested one installation in which the material was in small panels and well distributed, there being four small panels on each wall, each panel having an area of approximately $40 \mathrm{sq} \mathrm{ft}$. The ceiling was coffered, dividing it up into about 250 small squares, each having an area of a trifle over $2 \mathrm{sq} \mathrm{ft}$. Acoustical material having a 
coefficient of .95 for a $72 \mathrm{sq} \mathrm{ft}$ area, as measured in the reverberation room, was installed in these squares and panels.

From measurements made before and after the installation the average coefficient of sound absorption figures out to be approximately 1.2. At first thought this appears absurd, but if one refers to figure 13 and interpolates for a material having an absorption coefficient of .95 for a $72 \mathrm{sq} \mathrm{ft} \mathrm{sample,} \mathrm{one} \mathrm{would} \mathrm{get} \mathrm{an} \mathrm{absorption} \mathrm{coeffi-}$ cient of about 1.0 for a $40 \mathrm{sq} \mathrm{ft}$ sample and 1.4 for one of $2 \mathrm{sq} \mathrm{ft}$.

As about half the material was installed in the larger panels and the other half in the small squares, this would give 1.2 as the average. It should be realized that it was impossible to make these measurements with a great degree of precision and in checking the results it has been necessary to approximate, but it is very interesting to note

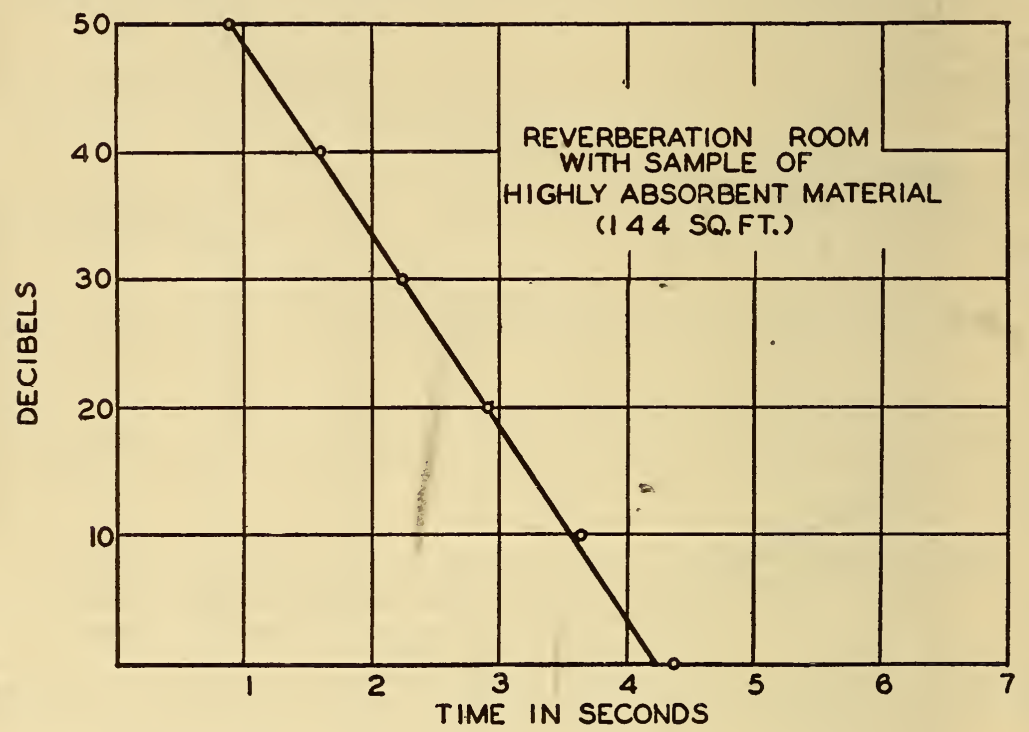

FIGURE 16.-Beginning of nonlogarithmic decay with increased area

that the results came out as one would have expected from the laboratory measurements. The reason for this agreement was probably the fact that the distribution of the absorbing material was such that the sound remained in a diffuse condition during the decay.

\section{CONCLUSION}

The greatest difficulty in designing a room so that it will be acoustically satisfactory is in determining, in advance of construction, the rate at which the sound energy will decay, as this depends upon the size of the panels of acoustical material and how these panels are distributed on the surfaces of the room. At the present time it is not. possible to predict this value accurately, no matter how accurately the coefficient is known. It should be possible, however, if proper studies are made on different types of installations, to establish an empirical relationship so as to obtain the rate of decay accurately enough for all practical purposes. 
As additional support to the above statement, we wish to quote from an article by Eyring which was printed in the January 1933 number of the Journal of the Acoustical Society of America:

Any kind of a reverberation formula used to calculate an absorption coefficient from a reverberation time may be used under the identical circumstances to calculate the reverberation time from the absorption data. In a word, it is simply the process of working forward through a mathematical procedure and then retracing the exact steps to the original data. Therefore, if the identical situation found in a chamber is also found in an auditorium, then any kind of formula could be used with success even though it describes a fictitious physical state.

In practice most reverberation chamber and auditorium situations are not identical. For this reason a reverberation time formula which truly describes the physical reality is much to be desired. In other words a true, not a fictitious, relationship between coefficients of absorption and reverberation time should be determined for as many different situations as possible.

Further work, both experimental and theoretical, is needed to put the computation of the acoustic properties of auditoriums on a satisfactory basis. It is hoped that this paper has indicated some of the directions which such investigations should take.

The author wishes to acknowledge the assistance of Catherine Miller Snyder who made a large part of the experimental observations.

Washington, June 26, 1934. 